\title{
MULTISPACECRAFT OBSERVATIONS OF SOLAR FLARE PARTICLES IN THE INNER HELIOSPHERE
}

\author{
G. Wibberenz \\ Institut für Experimentelle und Angewandte Physik, University of Kiel, D-24118 Kiel, \\ Germany \\ H. V. Cane ${ }^{1}$ \\ Astroparticle Physics Laboratory, NASA/GSFC, Greenbelt MD 20771
}

\begin{abstract}
For a number of impulsive solar particle events we examine variations of maximum intensities and times to maximum intensity as a function of longitude, using observations from the two Helios spacecraft and near the Earth. We find that electrons in the $\mathrm{MeV}$ range can be detected more than $80^{\circ}$ from the flare longitude, corresponding to a considerably wider "well connected" region than that ( $\sim 20^{\circ}$ half width) reported for ${ }^{3} \mathrm{He}$-rich impulsive solar events. This wide range and the decrease of peak intensities with increasing connection angle revive the concept of some propagation process in the low corona that has a diffusive nature. Delays to the intensity maximum are not systematically correlated with connection angles. We argue that interplanetary scattering parallel to the average interplanetary magnetic field, that varies with position in space, plays an important role in flare particle events. In a specific case variations of the time profiles with radial distance and with particle rigidity are used to quantitatively confirm spatial diffusion. For a few cases near the edges of the well connected region the very long times to maximum intensity might result from interplanetary lateral transport.
\end{abstract}

Subject headings: acceleration of particles-diffusion-Sun:flares-Sun:particle emission

\footnotetext{
${ }^{1}$ Also School of Mathematics and Physics, University of Tasmania, Hobart, Tasmania, Australia
} 


\section{INTRODUCTION}

Since the earliest spacecraft observations it has been apparent that there are two types of solar energetic particle (SEP) events. Lin (1974) described them as 'electron' and 'proton' events and noted that the former were associated with small flares. Later the two types of events were described as 'impulsive' and 'gradual' based on the observation that small events with low proton intensities are associated with short duration flares whereas larger events are associated with long duration flares (Cane et al. 1986). In addition to the low proton intensity impulsive SEP events are characterized by enhancements of heavy ions and in ${ }^{3} \mathrm{He}$ relative to ${ }^{4} \mathrm{He}$. Such abundance enhancements indicate that resonant processes are occurring and it is widely accepted that the particles are accelerated in flare heated plasma. In gradual events it is clear that shock acceleration plays an important role and most workers assume that essentially all the particles in gradual events are shock accelerated.

Although all gradual events are also associated with flares it is usually assumed that flare processes are not important because it is considered that such particles do not have access to open field lines (Reames 2002). However, new high sensitivity observations during cycle 23 have revealed that many large SEP events have abundances of Fe relative to $O$ and ${ }^{3} \mathrm{He} /{ }^{4} \mathrm{He}$ well above the so-called 'Reames values' that have been considered typical of gradual events. Several authors (e.g. von Rosenvinge et al. (2001); Cane et al. $(2003,2006)$; Laitinen et al. (2000)) have suggested that flares contribute in gradual SEP events. Others (e.g. Tylka et al. (2005)) argue against this possibility by suggesting that flare particles do not have access to an opening angle big enough to account for the distribution of source regions of gradual events with flare-like abundances. It is considered that flare particles seen near Earth only originate in source regions clustering around W50\%; Reames (1999) quotes a range of $\pm 20^{\circ}$ for small ${ }^{3} \mathrm{He}$-rich events with ${ }^{3} \mathrm{He} /{ }^{4} \mathrm{He}>0.1$. In contrast, gradual events with high $\mathrm{Fe} / \mathrm{O}$ occur in the range $\mathrm{E} 16^{\circ}$ to $\mathrm{W} 140^{\circ}$.

Unraveling the source regions of SEPs is especially difficult because the particles are observed remotely, usually at 1 AU and at a single spacecraft. An important source of information that has not yet been fully exploited is the data provided by particle experiments on the Helios spacecraft. Observations of the same SEP event from different locations provide important clues for understanding the complicated processes whereby particles are accelerated in the low corona and then propagate through the interplanetary medium. A surprising result from the Helios mission was that the biggest events seen at Earth were also seen at the Helios spacecraft even when they were at locations very remote from the longitude of the solar event. (Similarly, the largest events are seen by Ulysses even when it is near the

polar regions.) The current thinking attributes this widespread distribution of particles to acceleration at extensive coronal mass ejection (CME) driven shocks. 
In our three spacecraft study we investigate the longitudinal extent of flare acceleration by studying small (impulsive) events. Such a study has not been undertaken previously. Only a few studies have been undertaken combining data from the two Helios spacecraft with that taken near Earth (e.g. Beeck et al. (1987); Kallenrode et al., (1993); McGuire et al. (1983)). These earlier studies mainly considered gradual events in which shocks play a major role. Indeed Kallenrode et al., (1993) specifically addressed the efficiency of shock acceleration. We concentrated on electron observations but in most events protons were also seen at least at one location. At about $10 \mathrm{MeV}$ the proton intensities were typically two orders of magnitude below the $\sim 1 \mathrm{MeV}$ electron intensities; a deficit of protons relative to electrons is a characteristic of flare acceleration. For these events we do not expect any further acceleration beyond the initial injection close to the Sun and thus we can better understand particle propagation in the interplanetary medium. Note that in recent years the role of particle propagation in the interplanetary medium has often been ignored for SEP events under the assumption that the particles travel essentially scatter free, with a mean free path along the magnetic field $\left(\lambda_{\|}\right)$of approximately 1 AU (Reames 1999). Also ignored, indeed discarded, is the possibility of some transport process in the corona that enables particles to be observed from regions distant from a flare source. Acceleration at a shock obviates the need for such a process and so-called "coronal propagation" has barely been mentioned since the 1980's although it was a topic of intense interest in the preceding decades. A summary was presented by Kunow et al. (1991).

In this paper we examine variations of maximum intensities of flare particles, and times to maximum intensity, as a function of longitude using observations from Helios 1 and 2 and a near-Earth spacecraft. In order to compare intensities and rise times at different longitudes we needed to normalize the intensities to a fixed radial distance and adjust the times; the Helios orbits took them within $0.3 \mathrm{AU}$ of the Sun and at varying distances from the Sun-Earth line. We argue, based on the analysis of a specific event in Section 3, that these normalizations may be made using the predictions of a simple diffusion model. Section 4 illustrates the results for 19 flare events and they are discussed in Section 5.

\section{Data base}

Our study is based on electron events seen near Earth as well as on both Helios 1 and Helios 2. The electron channel E03 of the University of Kiel experiments on the two Helios spacecraft (see Kunow et al. (1977)) corresponded to a nominal energy range 0.3-0.8 MeV, based on the simple energy-range relationship in the detectors. An accurate Monte-Carlo calculation where large angle scattering of the electrons is taken into account (Bialk 1991) 
leads to a characteristic mean energy of this channel of $0.95 \mathrm{MeV}(\beta=v / c=0.94)$. For electron observations near Earth we used the JHU/APL experiment on IMP 8 and the GSFC experiment on ISEE 3. Based on a comparison of the electron observations when the Helios spacecraft were near Earth we determined that multiplying the $0.2-2.5 \mathrm{MeV}$ channel on IMP 8 by a factor of 0.45 and the $0.2-2 \mathrm{MeV}$ channel on ISEE 3 by 4.5 provides a good comparison with the nominal $0.3-0.8 \mathrm{MeV}$ channels on the Helios spacecraft.

\section{Interplanetary transport}

In order to make a proper comparison of intensities at different longitudes for the same event combining Helios and near Earth spacecraft an adjustment must be made for the fact that the Helios spacecraft varied in radial distance from the Sun. Thus we first discuss a unique situation that occurred shortly after the launch of Helios 2 when three spacecraft were located almost on the same field line but at different radial distances. As a result we find that the interplanetary transport can be described by spatial diffusion along the average heliospheric magnetic field.

Figure 1(a) shows the relative locations of IMP 8 and the Helios spacecraft on 1976 March 21. At the time there was an active region near $\mathrm{W} 33^{\circ}$ as indicated by the line marked "Flare". Helios 1 was at 0.36 AU, Helios 2 at 0.59 AU. The Archimedean spirals passing through the spacecraft indicate the field line connections back at the Sun taking into account the measured solar wind speeds at the spacecraft at about 13:00 UT. The foot points of the field lines were determined to be at about $W 43^{\circ}, W 38^{\circ}$ and $W 42^{\circ}$ for Helios 1, Helios 2, and IMP 8 respectively. The differences in solar wind speed at the respective positions lead to wider separation of the magnetic field spirals further out. The corresponding connection angles $\Delta$, defined as the difference between flare longitude and foot points, were $-10^{\circ},-5^{\circ}$, and $-9^{\circ}$. Connection angles are negative when the connection point is west of the flare.

Figure 1 (b) shows six hours of electron data for the event on 1976 March 21 with the type III burst starting around $t_{0}=12: 44$ solar release time (i.e. after subtraction of 8 minutes from the observed UT). The Helios data are 1 minute averages whereas the IMP 8 data are 5 minute averages. The variation of the time profiles with increasing radial distance from the Sun is a characteristic feature of diffusive transport. We use qualitative results from diffusive transport to estimate values of the radial mean free path $\lambda_{r}=\lambda_{\|} \cos ^{2} \phi$, with $\lambda_{\|}$ the scattering mean free path along the average IMF and $\phi$ the spiral angle. Let us assume a variation of $\lambda_{r}$ with radial distance $r$ as $\lambda_{r}(r)=\lambda_{0}\left(r / r_{0}\right)^{b}$, with $r_{0}=1$ AU. Therefore, $\lambda_{0}$ is the radial mean free path of the $\mathrm{MeV}$ electrons near $1 \mathrm{AU}$ for the event under study. Comparison of intensities at different radial distances from the Sun is a good means to 
determine the value of $b$ (see e.g. Hamilton (1977); Beeck et al. (1987)).

Based on a simple diffusion model with short injection and $\lambda_{r}(r)$ as specified above the results in Figure 1 can be interpreted as follows. The value of $b$ influences the decay phase of a solar event, with $I(t) \propto\left(t-t_{o}\right)^{-3 /(2-b)}$ at late times. A value of $b=0.5$, corresponding to a power law decay $\propto t^{-2}$, is in good agreement with the late phase of the electrons on Helios 1 (upper curve, Figure $1(\mathrm{~b})$ ). Note that a value of $b=0.5$ is in good agreement with the result $b=0.6 \pm 0.1$ based on multi-spacecraft observations from three different radial distances from the Sun (Beeck et al. (1987)).

For diffusive transport the peak intensities simply scale with the third power of the radial distance; this $r^{-3}$ variation is only determined by the geometry of the propagation medium, independent of the value of $b$. However the scaling does assume that a magnetic flux tube expands spherically symmetrically in three dimensions which is not strictly the case. The presence of fast and slow solar wind streams in combination with the rotation of the Sun leads to regions in space as a function of longitude where the expansion occurs more rapidly or more slowly with $r$, corresponding to compression and rarefaction regions adjacent to each other, see Nolte et al. (1977). We believe that this complication will not affect our results significantly. Thus for the 1976 March 21 event we expect a factor of $0.35^{3}=0.043$ difference in intensity between the locations of Helios 1 and IMP 8. In addition, the increase of the time-to-maximum with radial distance can be described by $t_{m} \propto r^{2-b}$. This means that for $b=0.5$ the profile at IMP 8 as compared to Helios 1 should be scaled by the factor of $0.35^{1.5}=0.207$ in the time axis. After background subtraction this scaling in intensity and time leads indeed to good agreement between the time profiles observed at IMP 8 and Helios 1 as may be seen in Figure 2. Note that both spacecraft are located practically on the same bundle of IMF field lines; the nominal connection longitudes differ only by $1^{\circ}$ (see above). One should therefore expect the same injection at the foot points of both spacecraft. For scatter-free transport the whole time profile at IMP 8 should shift merely by the difference in direct travel time, and the peak intensities by a factor of $0.35^{2}$ only. The evidence presented here is clearly in favor of diffusive transport.

The absolute value $\lambda_{0}$ of the (radial) mean free path for the $0.95 \mathrm{MeV}$ electrons on Helios 1 as determined from the observed value of $t_{m}$ of $0.52 \mathrm{hr}$ is estimated as $\lambda_{0}=0.046 \mathrm{AU}$. Figure 1 (c) shows the $2-4 \mathrm{MeV} / \mathrm{n}$ He ions (lower curve) in comparison with the $\mathrm{MeV}$ electrons (upper curve) at Helios 1 . The expected difference between the arrival of helium nuclei $(\beta=v / c=0.065)$ and electrons $(\beta=0.94)$ in the case of scatter-free transport along the spiral length of $0.36 \mathrm{AU}$ is $0.7 \mathrm{hr}$, in contrast to observations. An estimated time-tomaximum of $2.8 \mathrm{hr}$ for the He ions leads to $\lambda_{0}=0.104 \mathrm{AU}$.

It should be noted that the width as well as the time-to-maximum of a "diffusive look- 
ing" event are determined both by the length of injection near the Sun and the diffusion in interplanetary space. A separation of these two influences can be obtained by the simultaneous inspection of intensities and anisotropies during an event. Based on this method Kallenrode et al.; (1992) applied the full numerical solutions of the equation of focused diffusion to periods where the two Helios spacecraft were located at radial distances within $0.5 \mathrm{AU}$ from the Sun. For six solar electron events the radial mean free path $\lambda_{r}$ was determined to be in the range 0.02 to $0.15 \mathrm{AU}$. This is close to the consensus range obtained by Palmer (1982), as well as mean free paths reported by MaSung and Earl (1978) for observations near Earth. We note that profiles for the events discussed in the present paper are very similar to those examined by Kallenrode et al.; (1992). Our simple method when applied to the Kallenrode et al., (1992) events gives values similar to those derived using the full numerical solutions and, in particular, for the events with a short injection.

An extensive discussion by Bieber et al. (1994) confirms the range of electron mean free paths in the $\mathrm{MeV}$ range characteristic for finite interplanetary scattering and stresses two more important results based essentially on the Helios data and analysis: a rather small change of $\lambda$ with rigidity in the range relevant for $\mathrm{MeV}$ electrons and several $\mathrm{MeV} /$ nucleon ions, and a high degree of variability in $\lambda$ from one event to the other, occurring simultaneously over a large range of rigidities, see also the original results by Kallenrode (1993) and a more recent presentation including electrons of lower energy by Dröge (2003). These findings will be important for the subsequent discussion. In particular, for a given period of time we should expect a variation of the degree of interplanetary scattering with the longitudinal position of the observer.

Figure 3 shows another example of multiple small events observed close to the Sun. The time difference between subsequent events in the 1980 May 28 period is shorter than in the case of the 1976 March 21 series of events. As shown in the connection plot (upper panel), Helios 1 was in an ideal situation, $0.31 \mathrm{AU}$ from the Sun and at a connection angle of $\Delta=-6^{\circ}$, closely connected to the flare. In contrast, IMP 8 was connected by $\Delta=-46^{\circ}$. The difference between the two electron curves is mainly caused by the radial positions and the role of interplanetary scattering. Kallenrode and Wibberenz (1991) estimated the mean free path at Helios 1, applying the focused diffusion model, to be in the range 0.10 to $0.15 \mathrm{AU}$. At IMP 8 interplanetary scattering plays a much larger role. At the time of a subsequent injection the intensity of the previous event has hardly reached its maximum. For the IMP 8 position the radial mean free path of the electrons is estimated to be 0.03 AU. For the 2-4 $\mathrm{MeV} /$ nuc $\mathrm{He}$ the different injections are clearly identified at Helios 1 but only a very small and very broad increase was seen at IMP 8 and no separate injections. 


\section{STATISTICAL STUDY}

\subsection{Variations with Connection Angle}

For the period of December 1977 to early March 1980 (when the Helios 2 mission ended) we investigated all flare events well observed at the three spacecraft. Events were selected on the basis of being short in duration and being associated with type III bursts and short duration flares. These solar event characteristics select against events with associated interplanetary shocks (Cane et al. 1986). Events where an interplanetary shock was observed were specifically excluded. That the selected events were indeed flare events was supported by the low proton to electron ratios. Some events had to be excluded because of data gaps in the particle data on one or more spacecraft and the requirement of increases above background leading to clearly identified peak intensities on all spacecraft. The final list of 19 events is given in Table 1 . Characteristics of the associated flares include the date and time (start of the metric type III burst), $\mathrm{H} \alpha$ location and class, and X-ray class. In 10 cases a type II radio burst was observed and its duration is given. The table also contains the connection angle $\Delta$ for the three spacecraft and the radial distance for Helios 1 and 2 . It should be noted that for this data set in only five out of the 19 events were the Helios spacecraft within $0.7 \mathrm{AU}$.

Figure 4 shows data for the event of 1979 January 15. Panel (a) shows the location of the three spacecraft with respect to the flare longitude at $W 79^{\circ}$, with connection angles of $90^{\circ}$ (Helios 1), 40. (Helios 2) and $31^{\circ}$ (IMP 8). The radial positions of Helios 1 and 2 were $0.94 \mathrm{AU}$ and $0.96 \mathrm{AU}$, respectively. The amplitudes as shown in panel (b) vary with connection angle $\Delta$ in a characteristic way ie. they decrease with increasing $\Delta$. However the rise times and the times-to-maximum are not correlated with $\Delta$, i.e. the delays do not increase with the distance from the flare longitude, see more details in Section 4.2.

In the subsequent presentations the amplitudes (i.e. intensities at the times of the intensity maxima) are normalized to 1 AU according to the $r^{-3}$ variation discussed in Section 3. Figure 5 shows normalized intensities as a function of connection angle for the 19 events. Amplitudes obtained for the same event are marked by different symbols and colors and connected by straight lines. For the events presented in Figure 5a the highest intensities are generally observed on field lines closest to the flare longitudes.

It is usually assumed that the azimuthal distribution of flare particles is rather narrow $\left( \pm 20^{\circ}\right.$ based on the observation of ${ }^{3} \mathrm{He}$-rich events (Reames 1999)). Having this in mind the wide longitudinal range of $\mathrm{MeV}$ electrons observed in flare events in Figure 5 may seem surprising. Although the amplitude dependence on longitude varies from one event to the other, we recognize a general pattern which is rather flat around the flare position $(\Delta=0)$ 
and has a general steepening for increasing angular distance. Note that for all the data points in Figure 5 the amplitudes are above background, so that the total width of the "connection range" covers about $160^{\circ}$. To guide the eye a Gaussian distribution is included, with standard deviation $\sigma=30^{\circ}$. For moderate angular distances this variation would correspond to an e-folding angle of the order of $\Delta_{o}=28^{\circ}$, or a factor of 10 decrease over $64^{\circ}$.

We also find events where the amplitude variation with longitude is rather unusual, as shown in Figure 5b for six out of our list of 19 events. In all these cases the highest intensities are not found for the spacecraft with the smallest connection angle. A possible explanation in terms of deviations from the standard back mapping procedure based on a Parker spiral would require a rather drastic variation. We shift the discussion of the transport and escape features of the accelerated electrons to section 5, where we also discuss the implications of the different connection cones of electrons and ${ }^{3} \mathrm{He}$ ions.

\subsection{Temporal structures and interplanetary scattering.}

Visual inspection of the intensity time profiles observed at the three spacecraft positions indicates differences in the times to maxima of the $\mathrm{MeV}$ electrons, as was shown in Figure 4. In that case the three observers were located at nearly the same radial distance. Based on the discussion in Section 3 we take the time-to-maximum at a certain position as a rough indication for the strength of interplanetary scattering. In Figure 6 we present the estimated values of $t_{m}$ (in hours) as a function of the absolute value of the connection angle $\Delta$. The selection of entries was limited to spacecraft positions outside of $0.6 \mathrm{AU}$, for the following reason. In order to scale the observation of the time-to-maximum to $1 \mathrm{AU}$ the value of $b$, which characterizes the radial variation of $\lambda(r)$, is required. As discussed in Section $3, t_{m}$ scales with $r^{2-b}$, but the value of $b$ may vary from one event to the other. The scaling to the value of $t_{m}$ shown in Figure 6 was performed by using $b=0.5$. For the range $0<\mathrm{b}<1$ the uncertainty in the value of $t_{m}$ expected at $1 \mathrm{AU}$ is smaller than about $25 \%$ for $\mathrm{r}>0.6 \mathrm{AU}$ which still seems acceptable in view of the observed variations and the other uncertainties when estimating $t_{m}$ from the profiles. The error bars on some of the events with $t_{m}<5$ hrs represent the variations expected for this range in $b$.

The horizontal line in Figure 6 represents a time-to-maximum of $5 \mathrm{hrs}$. In terms of ordinary diffusive transport with $b=0.5$ this would correspond to a value of $\lambda_{o}=0.019$ AU. Most of the events in Figure 6 have times below $5 \mathrm{hrs}$, corresponding to $\lambda_{0}$ larger than $0.019 \mathrm{AU}$. The values cover the range $0.023<\lambda_{o}<0.22 \mathrm{AU}$, i.e. within the range which has been obtained previously and quoted in Section 2. Conversion to $\lambda_{\|}=\lambda_{r} / \cos ^{2} \phi$, with a spiral angle $\phi=45^{\circ}$ leads to the range $0.046<\lambda_{\|}<0.44 \mathrm{AU}$. 
The important result is that Figure 6 does not indicate a systematic variation of $t_{m}$ with connection angle $\Delta$. In particular, there is no increasing temporal delay near the outer edges of the connection region. (The four points above the horizontal line are discussed below). The non systematic scatter reflects the variation of the mean free path from one location to the other, related to different scattering strength in the interplanetary medium. We note that this result appears to conflict with the work of Schellert et al. (1981) in which at large angular distances a systematic increase of $t_{m}$ with $\Delta$ was found. However that study did not restrict itself to flare events and the majority of the events were gradual. In these cases an extended interplanetary shock may have been responsible for the delayed intensities.

The relation between the onset time $t_{1}$ of an event and the time-to-maximum $t_{m}$ is shown in Figure 7 . The uncertainties in $t_{1}$ correspond to the use of 15 minute averaged data. The tendency of later onsets with increasing $t_{m}$ reflects the situation that in the case of strong scattering (large $t_{m}$ ) the intensity rises very slowly, leading to a later increase above background, see the results presented by Kallenrode and Wibberenz (1990).

\subsection{Lateral Transport.}

There are four events in Figure 6 where the values of $t_{m}$ are particularly large. We also added to Figure 6 a result for an impulsive event on 1980 May 12 which was observed at Helios 1 (at a radial distance of $0.5 \mathrm{AU}$ ) and ISEE 3 (Helios 2 was no longer in operation). This event is indicated with an asterisk. For the position of ISEE-3 at $\Delta=46^{\circ}$ the estimated $t_{m}$-value was $9 \pm 1$ hours, which would have corresponded to $\lambda_{o}=0.011 \mathrm{AU}$ in case of spatial diffusion along the average IMF and $b=0.5$. Similarly, for the event of 1980 March 2 Helios 1 was located at $0.98 \mathrm{AU}$ and at an angular distance of $91^{\circ}$. The observed $t_{m}=7.6 \pm 1$ hours would have corresponded to a mean free path of $\lambda_{0}=0.013 \mathrm{AU}$. The electron profiles for the two events just described are shown in Figure 8. (There are multiple events on 1980 May 12 from the same active region; we have considered the first and most intense one.) Note that there are four events shown in Figure 6 where the delay is within 5 hours even with $\Delta>70^{\circ}$ again indicating the variability in interplanetary propagation from event to event. There are also examples of events not seen at all for such poor connection. In these cases we cannot distinguish whether the intensities are below background or that no transport occurs at all up to this distance.

We believe it to be rather unlikely that the delays longer than 6 hours can be related to parallel interplanetary scattering since the estimated values of the mean free path are unusually small, smaller than reported in the literature. Therefore, we suggest the interpretation that the delay is related to transport perpendicular to the average magnetic field. A 
characteristic value for the perpendicular diffusion coefficient, based on Jovian electrons in the $\mathrm{MeV}$ range, was given by Ferrando (1997) as $K_{\perp} \sim 8 \times 10^{20} \mathrm{~cm}^{2} \mathrm{~s}^{-1}$. This value is in good agreement within the range obtained from Jovian electrons according to Palmer (1982). In the case of two-dimensional lateral diffusion the resulting time constant near $1 \mathrm{AU}$ up to maximum intensity would be $t_{d}=\left(r^{2} \theta^{2}\right) /\left(4 K_{\perp}\right)$, resulting in $t_{d}=7$ hours for an angular distance of $\theta=30^{\circ}$. Such a value of $\theta$ is just an estimate for the angular distance to be traversed between the region of good connection with observed high intensities and lateral transport to the distant observer. This estimate is in qualitative agreement with the four largest $t_{m}$-values between 6 and 9 hours obtained in Figure 6.

A lateral diffusion process was also suggested to explain several observations at high latitudes using Ulysses observations (e.g. Zhang et al. (2001); Dalla et al. (2003)). However, direct comparisons with our results are difficult, in particular because of the presence of large interplanetary shocks in the Ulysses events. Cross-field transport has also been suggested for solar particles in the ecliptic. Cane and Erickson (2003) studied the delay times of very low frequency type III radio bursts as well as of $38-53 \mathrm{keV}$ electrons and 11-23 MeV protons and found that the delays increased with increasing angular distance from the flare region. They concluded that for the very slow events interplanetary cross-field transport occurs.

\section{Discussion}

\subsection{General.}

We have studied the behavior of $\mathrm{MeV}$ electrons in a series of impulsive, flare-related events which were observed simultaneously at three different positions. The events appear distributed in angular distances $\Delta$ from the flare over a range of the order of $\pm 80^{\circ}$. Within the same event, we generally observe a systematic decrease of amplitudes with increasing $\Delta$ (Figure 5a). For the bulk of data, the temporal structures can be interpreted in terms of interplanetary scattering, corresponding to radial mean free paths $\lambda_{\circ}$ in the range 0.023 to $0.22 \mathrm{AU}$. No systematic increase of the time-to-maximum with increasing lateral distance can be found (Figure 6). In summary, accelerated electrons appear in the low corona over a large range of longitudes nearly simultaneously. However, the total number of particles observed in space in general decreases systematically with angular distance from the flare. The question is whether these features can be related to the acceleration process itself or whether a fast transport of electrons in the corona occurs over distances up to $80^{\circ}$. Only for very few cases are there long delays observed in space, which we relate tentatively to perpendicular transport in the interplanetary medium (see section 4.3). One obvious result, however, is that the extent of the well connected region for energetic electrons of the order 
of $\pm 80^{\circ}$ is markedly larger than for the He3-rich events of the order of $\pm 20^{\circ}$.

\subsection{Interplanetary scattering.}

In the inner solar system the focused transport equation as introduced by Roelof (1969) takes the combination of pitch angle scattering and focusing properly into account. As a measure for the degree of interplanetary scattering one generally uses the mean free path $\lambda_{\|}$. It represents a suitable average over all pitch angle scattering coefficients as discussed by Hasselmann and Wibberenz (1970) and Earl (1974). It should be realized that this value of $\lambda_{\|}$is not identical with the distance which each individual particle travels along the IMF before it is scattered back. Early in a solar particle event the backscattering of particles may be very small. This leads to the "beamed" arrival of the first group of particles. In a later phase spatial diffusion sets in, in accordance with the difference between the "coherent peak" and the "diffusive wake" in the terminology of Earl (1976). An example can be seen in the paper by Ho et al. (2001). They describe the onset and rising phase of ${ }^{3} \mathrm{He}$ particles in their Figure 1 in terms of scatter-free propagation times. Nevertheless, the total duration of the event was of the order of half a day, and the event should therefore be discussed in terms of spatial diffusion combined with focusing. It is well established (see e.g. Dröge (2003)) that for a given solar flare event the mean free path of electrons increases with decreasing rigidity below a broad minimum in the MV rigidity range. When this effect is combined with the variability of the mean free path from one event to the other (see Kallenrode (1993), Bieber et al. (1994), Dröge (2003)), one expects a high (low) probability for weak scattering for $\mathrm{keV}(\mathrm{MeV})$ electrons. The term "scatter-free ${ }^{3} \mathrm{He}$-events" should also be used with some care. The average mean free path of ${ }^{3} \mathrm{He}$ in the $\mathrm{MeV} /$ nucleon range is only slightly larger than that of $\mathrm{MeV}$ electrons. Therefore, we expect the same pattern of non systematic variation of the mean free path with longitude for ${ }^{3} \mathrm{He}$ as for $\mathrm{MeV}$ electrons. It is quite possible that ${ }^{3} \mathrm{He}$-rich events are preferentially detected under conditions of nearly scatter-free transport because they are then more prominent above the background. The difference between fast and slow rising ${ }^{3} \mathrm{He}$-rich events was mentioned by Mason et al. (2002), and they note that ${ }^{3} \mathrm{He}$-rich events with gradual profiles may have been missed in the earlier work because of the combination of lower sensitivity and resolution in comparison with the ACE instrumentation.

In Section 4.2 the diffusive approximation was used to take the time-to-maximum $t_{m}$ (Figure 6) as an indication for the degree of scattering of $\mathrm{MeV}$ electrons. The values based on the simple estimates cover the range $0.023<\lambda_{0}<0.22 \mathrm{AU}$, in good agreement with the range obtained from the study of Helios electron events by using the focused transport equation (see Kallenrode et al., (1992)). The corresponding values of the parallel mean free 
paths of $0.046<\lambda_{\|}<0.44 \mathrm{AU}$ also correspond to the results for five out of six electron events studied by Dröge (2000). The values of $t_{m}$ in Figure 6 below the critical limit of 5 hours are distributed at random with connection angle. This indicates that the underlying temporal structure can be interpreted in terms of variable interplanetary scattering, and we envisage fast transport of the accelerated particles near the Sun.

When considering average values it is rather encouraging that in a broad sense one may use the same set of diffusion coefficients in the equatorial regions at $1 \mathrm{AU}$ for the study of solar and Jovian electrons. This can be seen by comparison with the results of Ferreira et al., (2001). As one basis for their fully three-dimensional model of the heliospheric modulation of galactic and Jovian electrons they use a value of the parallel mean free path near 1 AU of the order of 0.1 AU based on solar particle observations contained in Bieber et al. (1994). The situation with respect to the perpendicular diffusion is more complex for several reasons. Results from Jovian electrons were mostly obtained from the spatial regions between 1 and $5 \mathrm{AU}$; there exist two different values of $K_{\perp}$ in the radial and in the latitudinal direction; CIRs provide an effective impediment to cross-field diffusion; to our knowledge no generally accepted theoretical estimate for the size of the perpendicular diffusion seems to exist, in particular not in the MV range of rigidities, see the discussion in Giacalone and Jokipii (1999). A suggested "consensus value" by Palmer (1982) of the order of $K_{\perp} \sim 10^{21} \mathrm{~cm}^{2} \mathrm{~s}^{-1}$ leads to a time constant which is qualitatively consistent with the cases for lateral transport which we considered in section 4.3.

\subsection{The width of the well connected region}

Since impulsive events are often identified by the ${ }^{3} \mathrm{He}$-richness of the accelerated ions, the quoted "connection region" on the solar disk for impulsive events is usually based on ${ }^{3}$ He-observations, with a characteristic extent of the order of $\pm 20^{\circ}$. Before turning to a comparison with the $\mathrm{MeV}$ electron data in Section 4 we want to briefly describe the behavior of electrons of much lower energy. Lin (1970) discusses the acceleration and escape of $10-100 \mathrm{keV}$ electrons. He finds for a large active region that electrons have direct access to interplanetary field lines in an "open cone" with an extent of approximately $\pm 35^{\circ}$ in longitude. The width of this open cone - which may involve acceleration directly onto the open field lines - probably varies from one active region to another.

We are now faced with the result of different angular extents of three phenomena related to impulsive solar flares, increasing from (a) ${ }^{3} \mathrm{He}$-rich events over (b) $>45 \mathrm{keV}$ electrons observed directly in space to (c) $\sim \mathrm{MeV}$ electrons presented in this paper. It is not clear whether these differences are related to properties of the acceleration process and/or the escape and 
transport properties of the three particle types. A narrow distribution for the connection region of ${ }^{3} \mathrm{He}$-rich events may not be too surprising in terms of suggested acceleration models. Requisites for ${ }^{3} \mathrm{He}$ acceleration include a hot plasma of the solar flare acceleration region, low energy electrons streaming away from the Sun, and propagating plasma and/or MHD waves (for discussion see Temerin and Roth (1992); Miller (1998)). Therefore, it ought to be possible to derive from the acceleration models requirements for the lateral extent over which the preferential acceleration of ${ }^{3} \mathrm{He}$ occurs, e.g. related to the existence of obliquely propagating waves.

Reames (1999) notes that acceleration of ${ }^{3} \mathrm{He}$ seems to be a direct, inescapable consequence of the electrons streaming in flares. Based on the ISEE 3 results, Reames et al. (1985) had found that impulsive $0.2-2 \mathrm{MeV}$ electron events accompany all ${ }^{3} \mathrm{He}-$ rich event periods, but that large clear electron events are not always accompanied by detectable ${ }^{3} \mathrm{He}$. In contrast, Ho et al. (2001) using ACE observations find a highly statistically significant association between the occurrence of ${ }^{3} \mathrm{He}-$ rich ion events and of $38-53 \mathrm{keV}$ impulsive electron events, though the maximum electron intensity appears statistically uncorrelated with the ${ }^{3} \mathrm{He} / \mathrm{He} 4$ ratio (at $0.4-2.0 \mathrm{MeV} / \mathrm{nuc}$ ). A preliminary conclusion seems to be that though the existence of low energy electrons is a necessary condition for the acceleration of ${ }^{3} \mathrm{He}$, it is not a sufficient one. This is in principal agreement with the wider cone of the $>40 \mathrm{keV}$ electrons in comparison with ${ }^{3} \mathrm{He}$.

Now the question arises automatically whether the wider extents for the two electron energy groups can also be directly related to the acceleration process. In the "open cone" idea the $>40 \mathrm{keV}$ electrons are also accelerated at the flare site, but a system of open field lines extends from that location and spans a large longitudinal distance further away from the Sun (Lin 1970). Such a large scale regular structure of the coronal magnetic fields seems very unlikely, and this kind of a "direct access" looks even more improbable for $\pm 80^{\circ}$ angular extent observed for the $\sim \mathrm{MeV}$ electrons.

Of course the observation of solar particles over large angular extents is a puzzle that has been discussed for many decades. In recent years the prompt arrival of particles from poorly connected regions in the largest gradual events is usually attributed to an extended shock that accelerated particles directly on the observers field line. However, with respect to the $\sim \mathrm{MeV}$ electrons we have specifically selected impulsive flare events in which shocks are generally not considered to play a role (see the discussion in section 4.1). If shocks are not involved then the most likely interpretation is the combination of transport and escape mechanisms of energetic electrons from the active region in systems of magnetic loops. The lateral transport of energetic particles could occur along magnetic loops (see e.g. Newkirk and Wentzel (1978)) and reconnection could channel particles to open field lines. 
Formerly, these processes had been combined into a model of coronal diffusion, as suggested originally by Reid (1964) and Axford (1965). Such a model lends itself naturally to a nearly exponential variation of maximum intensities with angular distance, and the arrival time at a position in space being determined by interplanetary scattering for small coronal transport times (see Wibberenz et al. (1989) for details). The finite extent of the "fast propagation region" should be determined by the properties of the low corona at the time of a specific event and may be limited at its outer edges e.g. by unipolar regions with no loops crossing.

Above we had considered it unlikely that the angular extent of the acceleration process for the three particle types is so drastically different that it would directly cause the different angular extents. Should a coronal transport process exist, as suggested above for the energetic electrons, it is conceivable that it also operates for the other two particle types, but with different efficiencies. Qualitatively, the coronal transport times would scale with the inverse of the particle velocities which are $\beta=0.94 ; 0.37 ; 0.046$ for $\sim \mathrm{MeV}$ electrons, $40 \mathrm{keV}$ electrons, and $\sim \mathrm{MeV} /$ nuc $^{3} \mathrm{He}$ ions, respectively. For long coronal transport times (as in the case of low energy ${ }^{3} \mathrm{He}$ ions) the bulk of the particles could escape on open field lines before they get to large connection angles. The resulting intensity decrease with lateral distance from the flare position would eventually be so steep that it would be difficult to resolve low intensity events remote from the direct acceleration site. This interpretation of the different behavior of the high energy electrons and the ${ }^{3} \mathrm{He}$ ions corona would then not require the introduction of two different classes of impulsive events as suggested by Kallenrode (2003).

Although in our study we specifically excluded events that were accompanied by interplanetary shocks observed locally at one of the positions, we mention that about half of our events had associated metric type II bursts (related to coronal shocks). We also note that based on recent results (Cane, in preparation, 2006) it is likely that most of the events in this multispacecraft study had associated CMEs. Even if all or most of these CMEs caused (coronal) shocks we consider efficient acceleration processes unlikely. These possible shocks would be relatively weak since they did not survive to the interplanetary medium. In any case the events which we studied are proton poor, a characteristic difference from shock related events. Finally, one would not expect that a shock would inject particles only for the very short injections inferred from the modeling of events of the type under study (see. e.g. Kallenrode et al., (1992)) However, the existence of expanding CMEs, that change the magnetic field structure of the solar corona, might help to explain deviations from a regular amplitude variation as observed in Figure 5b.

Although we can not determine why or how flare electrons fill such a large angular extent our study suggests that the very narrow cone of emission quoted for ${ }^{3} \mathrm{He}-$-rich events greatly underestimates the cone of emission for impulsive flare particles in general. Thus a 
contribution of flare particles in major events cannot be ruled out on the basis of arguments using the distribution of ${ }^{3} \mathrm{He}$-rich events observed near Earth. Further thorough analysis of available sets of energetic particle data is encouraged. Results from the upcoming STEREO and Sentinel missions will provide valuable additional information.

Finally we mention a few additional studies.

1. An analysis of the connection ranges for different species of particles from individual active regions during their passage over the solar disk should be undertaken. We have found that the ratio of electrons to ions can vary from one event to the next from the same active region. Problems include their highly variable spatial extent and the complexity of flaring loop structures. It is observed that features of such regions change with time. This includes the transition from open to closed structures as can be inferred when an active region continues to exhibit $\mathrm{H} \alpha$ and $\mathrm{X}$-ray flaring but without associated type III bursts.

2. A more detailed study would use the more sensitive instruments now available, including also ${ }^{4} \mathrm{He}$ ions and protons. So far it is not at all clear whether or not ${ }^{4} \mathrm{He}$ ions in general follow the same longitudinal variation as either electrons or ${ }^{3} \mathrm{He}$ ions.

\section{Summary}

For a group of impulsive solar events observed at three different longitudes we find prompt detectable electron increases in the $\mathrm{MeV}$ energy range beyond longitudinal distances of $80^{\circ}$ from the $\mathrm{H} \alpha$ flare position. This extent of the well connected region is considerably wider than observed previously for ${ }^{3} \mathrm{He}$-rich events. The decrease of peak intensity with connection angle suggests lateral transport via magnetic loops in the low corona. Times to reach maximum intensity are not systematically related to the connection angle. This suggests that interplanetary scattering is important, in particular since the short injections expected for the small events in this study should not contribute significantly to the temporal width observed in space: For a few cases near the edges of the well connected region the long times to maximum intensity are possibly a result of interplanetary lateral transport. Lateral transport has also been suggested to explain long delays to particle onset at high solar latitudes as observed by Ulysses.

This work was partially funded by a NASA contract with USRA. It is with pleasure that G.W. acknowledges the use of the Helios data which have been obtained and analyzed 
decades ago and the expertise of the space science team of the University of Kiel, in particular H. Kunow, R. Müller-Mellin, G. Green, and M.-B. Kallenrode. We thank the referee for constructive criticism and helpful comments and Ian Richardson for software assistance.

\section{REFERENCES}

Axford, W. I. 1965, Planet. Space Sci. 13, 1301

Beeck, J. et al. 1987, ApJ, 322, 1052

Bialk, M. 1991, Diplomarbeit, University of Kiel

Bieber, J. et al. 1994, ApJ, 420, 294

Cane, H.V. and Erickson, W. C. 2003, J. Geophys. Res., 108 (A5), 1203, doi:10.1029/2002JA009488

Cane, H. V., McGuire, R. E., and von Rosenvinge, T. T. 1986, ApJ, 301, 448

Cane, H. V., von Rosenvinge, T. T., Cohen, C. M. S., and R. A. Mewaldt 2003, Geophys. Res. Lett., 30(12), 8017, doi:10.1029/2002GRL016580

Cane, H. V., Mewaldt, R. A., Cohen, C. M. S., and von Rosenvinge, T. T. 2006, J. Geophys. Res., in press.

Dalla, S. et al. 2003, Annales Geophys., 21, 1367

Dröge, W. 2000, ApJ, 537, 1073

Dröge, W. 2003, ApJ, 589, 1027

Earl, J. A. 1974, ApJ, 193, 231

Earl, J. A. 1976, ApJ, 205, 900

Ferrando, P. 1997, Adv. Space Res. 19, 905

Ferreira, S. E. S., et al. 2001, J. Geophys. Res.106, 24,979 (A11), 20012JA000082

Giacalone, J., and Jokipii, J.R. 1999, ApJ, 520, 204.

Hamilton, D. C. 1977, J. Geophys. Res., 82, 2157

Hasselmann, K. and Wibberenz, G. 1970, ApJ, 162, 1049 
Ho, G. C. et al. 2001, ApJ, 552, 863

Kallenrode, M.-B 1993, J. Geophys. Res., 98, 19037

Kallenrode, M.-B., 2003, J. of Physics G: Nuclear Physics, 29, 965

Kallenrode, M.-B., and Wibberenz, G. 1990 Proc. 21st Int. Cosmic Ray Conf., 5, 229

Kallenrode, M.-B., and Wibberenz, G. 1991, ApJ, 376, 787

Kallenrode, M.-B., Wibberenz, G. and Hucke, S. 1992, ApJ, 394, 351

Kallenrode, M.-B., et al. 1993, Solar Phys., 147, 377

Knoll, R. et al.1978, IEEE Trans. Geosc. Electronics, GE-16, 199

Kunow, H. et al. 1977, J. Geophys., 42, 615

Kunow, H. et al. 1991, Physics of the Inner Heliosphere, 2., edited by R. Schwenn and E. Marsch, Springer, Berlin, pp. 243-342

Laitinen, T. et al. 2000, Astron. Astrophys., 360, 729

Lin, R. P. 1970, Solar Phys., 15, 453

Lin, R. P. 1974, Space Sci. Rev., 16, 189

Mason, G. M., et al. 2002, ApJ, 574, 1039

MaSung, L. S. and Earl, J. A. 1978, ApJ, 222, 1080

McGuire, R. E., Van Hollebeke, M. A. I., and Lal, N. 1983, Proc. 18th Int. Cosmic Ray Conf., 10, 357

Miller, J. A. 1998, Space Sci. Rev., 86, 79

Newkirk, G., Jr., and Wentzel, D.G. 1978, J. Geophys. Res., 83, 2009

Nolte, J. T., Krieger, A. S., Roelof, E. C., and Gold, R. E. 1977, Solar Phys., 51, 459

Palmer, I. D. 1982, Rev. Geophys. Space Phys., 20, 335

Reames, D. V., von Rosenvinge, T. T., and Lin, R. P. 1985, ApJ, 292, 716

Reames, D. V. 1999 Space Sci. Rev., 90, 413

Reames, D. V. 2002 ApJ, 571, L63 
Reid, G. C. 1964, J. Geophys. Res., 69, 2659

Roelof, E. C. 1969, Lectures in High Energy Astrophysics, ed. H. Ogelmann and J. R. Wayland (SP-199: Washington: GPO), 111

Schellert, G., Wibberenz, G., and Kunow, H. 1981, Proc. 19th Int. Cosmic Ray Conf., 4, 305

Steinberg, J. L., Hoang, S., and Dulk, G. A. 1985, Astron. Astrophys., 150, 205

Temerin, M. and Roth, I. 1992, ApJ, 391, L105

Tylka, A. J., Cohen, C. M. S., Dietrich, W. F., et al. 2005, ApJ, 625, 474

von Rosenvinge, T. T., et al., 2001 in Solar and Galactic Composition, ed. R. F. WimmerSchweingruber; pp. 343-348, American Institute of Physics, Woodbury, NY

Wibberenz, G., et al. 1989, Solar Phys., 124, 353

Zhang, M. 2003, J. Geophys. Res., 108 (A4), SSH4-1, DOI 10.1029/2002JA009531 
Table 1. THREE SPACECRAFT EVENTS

\begin{tabular}{|c|c|c|c|c|c|c|c|c|c|c|c|}
\hline \multirow[t]{2}{*}{ Event ${ }^{a}$} & \multirow[t]{2}{*}{ Date } & \multirow{2}{*}{$\begin{array}{l}\text { Time }^{\mathrm{b}} \\
\text { U.T. }\end{array}$} & \multicolumn{3}{|c|}{ Flare } & \multirow{2}{*}{$\begin{array}{l}\text { Type II } \\
\text { mins. }\end{array}$} & \multicolumn{3}{|c|}{$\Delta^{\mathrm{e}}$} & \multicolumn{2}{|c|}{ Distance (AU) } \\
\hline & & & Location & $\begin{array}{c}\mathrm{H} \alpha \\
\text { Class }^{c}\end{array}$ & $\begin{array}{l}\text { Xray } \\
\text { Peak }\end{array}$ & & $\mathrm{H1}$ & $\mathrm{H} 2$ & I8 & $\mathrm{H1}$ & $\mathrm{H} 2$ \\
\hline $1^{*}$ & $1977 \mathrm{Dec} 06$ & 1934 & S18 W18 & $-B$ & M3 & 16 & -2 & -34 & -50 & 0.79 & 0.75 \\
\hline 2 & 1977 Dec 24 & 1303 & S27 W43 & $\mathrm{dg}$ & $\mathrm{C} 2$ & 5 & 24 & -15 & -13 & 0.91 & 0.89 \\
\hline $3^{*}$ & $1978 \operatorname{Jan} 07$ & 0305 & S23 W65 & $-\mathrm{N}$ & $\mathrm{C} 4$ & - & 58 & 33 & 19 & 0.96 & 0.95 \\
\hline 4 & $1978 \mathrm{Feb} 11$ & 0816 & N11 E10 & $1 \mathrm{~N}$ & $\mathrm{C} 5$ & - & -21 & -60 & -78 & 0.96 & 0.97 \\
\hline 5 & 1978 Feb 11 & 1425 & N14 E06 & $1 \mathrm{~B}$ & $\mathrm{C} 9$ & - & -16 & -59 & -75 & 0.96 & 0.96 \\
\hline $6^{*}$ & $1978 \mathrm{Feb} 25$ & 1449 & N19 W21 & $1 B$ & $\mathrm{C} 4$ & 32 & 38 & 11 & -47 & 0.9 & 0.91 \\
\hline 7 & 1978 Apr 07 & 2015 & N20 W05 & $\mathrm{dg}$ & M1 & 4 & 15 & -19 & -46 & 0.52 & 0.54 \\
\hline 8 & 1978 Nov 08 & 1751 & N18 E12 & $1 B$ & C5 & - & 98 & 35 & -62 & 0.32 & 0.33 \\
\hline 9 & 1979 Jan 15 & 1335 & S18 W79 & $1 N$ & M2 & - & 90 & 40 & 31 & 0.94 & 0.96 \\
\hline 10 & $1979 \mathrm{Feb} 05$ & 1906 & N07 W35 & $-B$ & $\mathrm{dg}$ & - & 28 & -1 & -36 & 0.98 & 0.98 \\
\hline 11 & $1979 \mathrm{Feb} 18$ & 1637 & N17 W14 & 1B & $\mathrm{dg}$ & - & 36 & -1 & -28 & 0.97 & 0.96 \\
\hline $12^{*}$ & 1979 Apr 14 & 1440 & N04 E08 & 1B & M2 & 13 & 14 & -15 & -69 & 0.63 & 0.54 \\
\hline 13 & 1979 Apr 15 & 2008 & N04 W06 & $1 B$ & $\mathrm{dg}$ & 13 & 45 & -9 & -34 & 0.61 & 0.52 \\
\hline 14 & 1979 Apr 16 & 2345 & N05 W21 & $-B$ & C3 & - & 51 & -3 & -27 & 0.6 & 0.51 \\
\hline 15 & $1980 \operatorname{Jan} 16$ & 2036 & N27 W77 & $-F$ & $\mathrm{C} 6$ & 10 & 85 & 47 & 29 & 0.87 & 0.93 \\
\hline 16 & $1980 \mathrm{Feb} 08$ & 0905 & N13 W79 & $1 \mathrm{~B}$ & $\mathrm{X} 2$ & 9 & 82 & 57 & 27 & 0.96 & 0.98 \\
\hline $17^{*}$ & $1980 \mathrm{Feb} 11$ & 2035 & N10 W33 & $-F$ & M6 & 8 & 41 & -9 & -24 & 0.97 & 0.98 \\
\hline 18 & $1980 \mathrm{Feb} 12$ & 0733 & N10 W38 & $1 \mathrm{~B}$ & $\mathrm{C} 2$ & - & 41 & -4 & -24 & 0.97 & 0.98 \\
\hline $19^{*}$ & 1980 Mar 02 & 1635 & S28 W71 & $-B$ & M2 & 11 & 91 & 57 & -3 & 0.98 & 0.94 \\
\hline
\end{tabular}

${ }^{a}$ Events with $*$ have maximum intensity not at best connected spacecraft

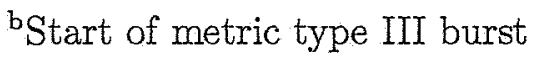

'‘dg' means data gap

'Type II duration. '-' no Type II

ePositive means footpoint is to the east of the flare 


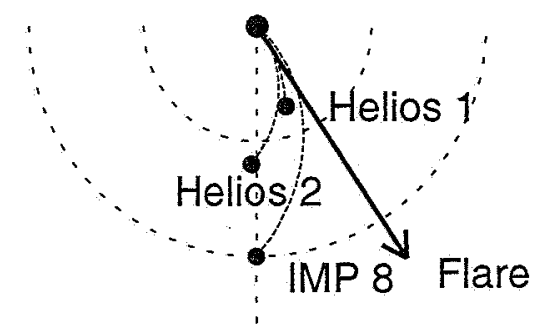

(a)

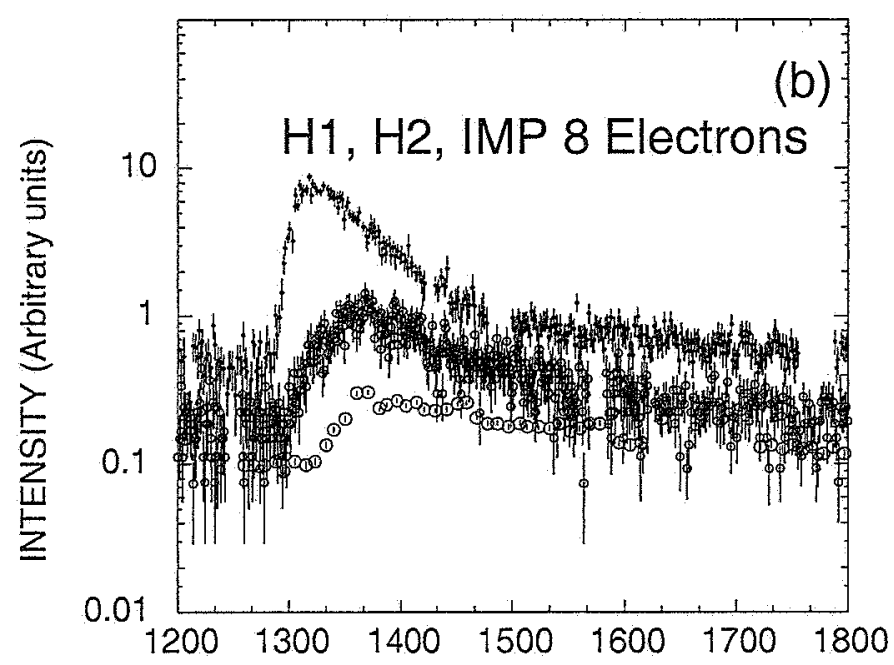

1976 March 21

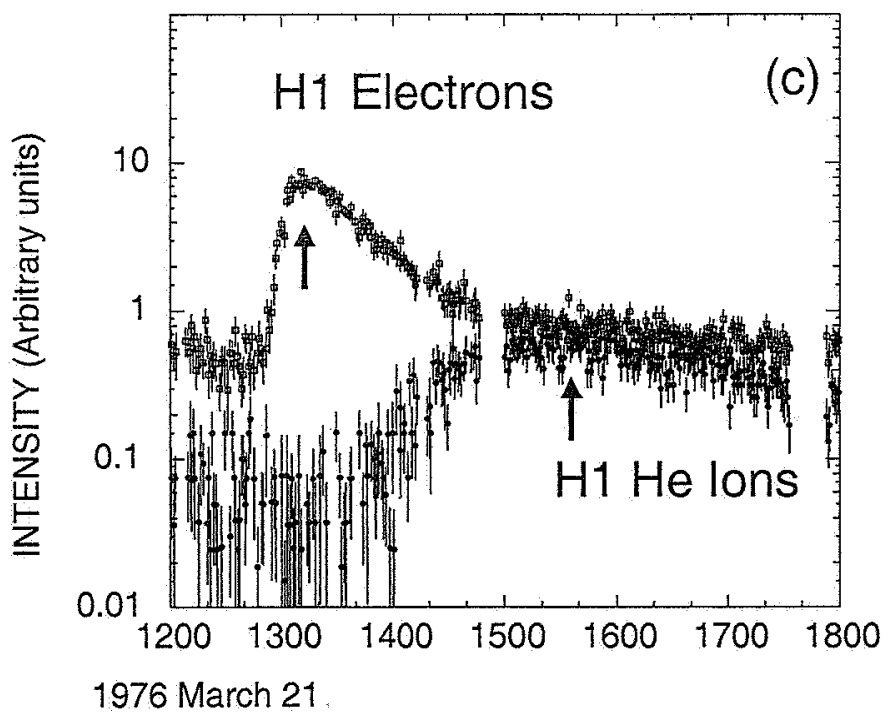

Fig. 1.- (a) Connection plot for the W33 flare of 1976 March 21, 1307 UT. Smooth spirals are drawn according to the measured solar wind speeds at the three spacecraft positions. (b) Electron intensities at the three different radial positions. (c) Time profiles of electrons and helium ions $(2-4 \mathrm{MeV} / \mathrm{n})$ on Helios at a position of $0.35 \mathrm{AU}$. The two arrows indicate the values $t_{m}$ for the peak intensities. The large difference between these two times is a clear indication for diffusive transport (see text). 


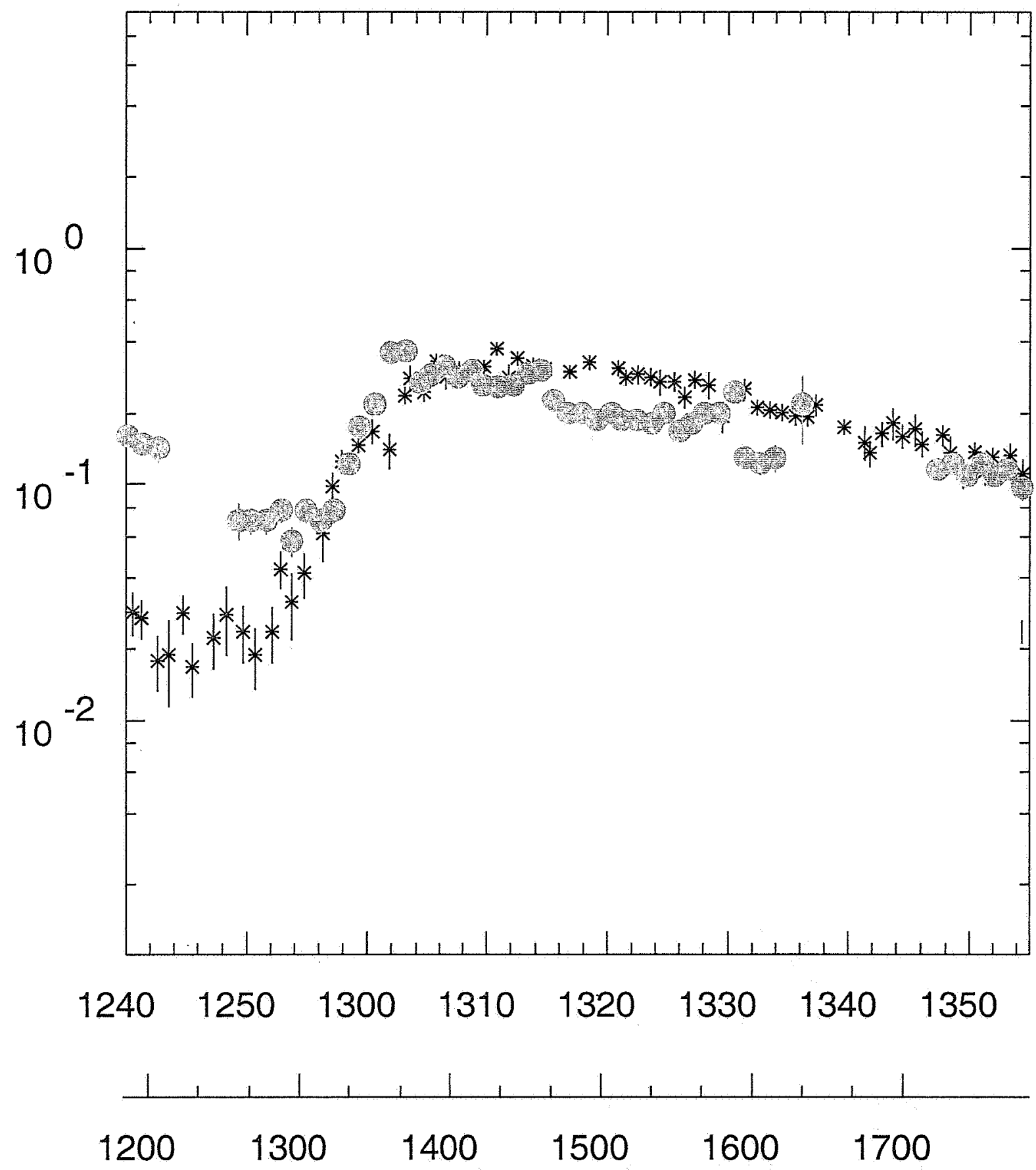

\section{March 21}

Fig. 2.- Electron intensities at Helios 1 (black curve) and at IMP 8 (grey curve). The Helios1 data have been corrected to 1 AU taking into account an $r^{-3}$-scaling in intensity, an $r^{1.5}$-scaling in time, corresponding to the two separate time axes at the bottom. The lower axis is for IMP 8 at the greater radial distance. Note the overall good agreement between the two profiles, apart from the later onset above the (higher) background at IMP 8. 

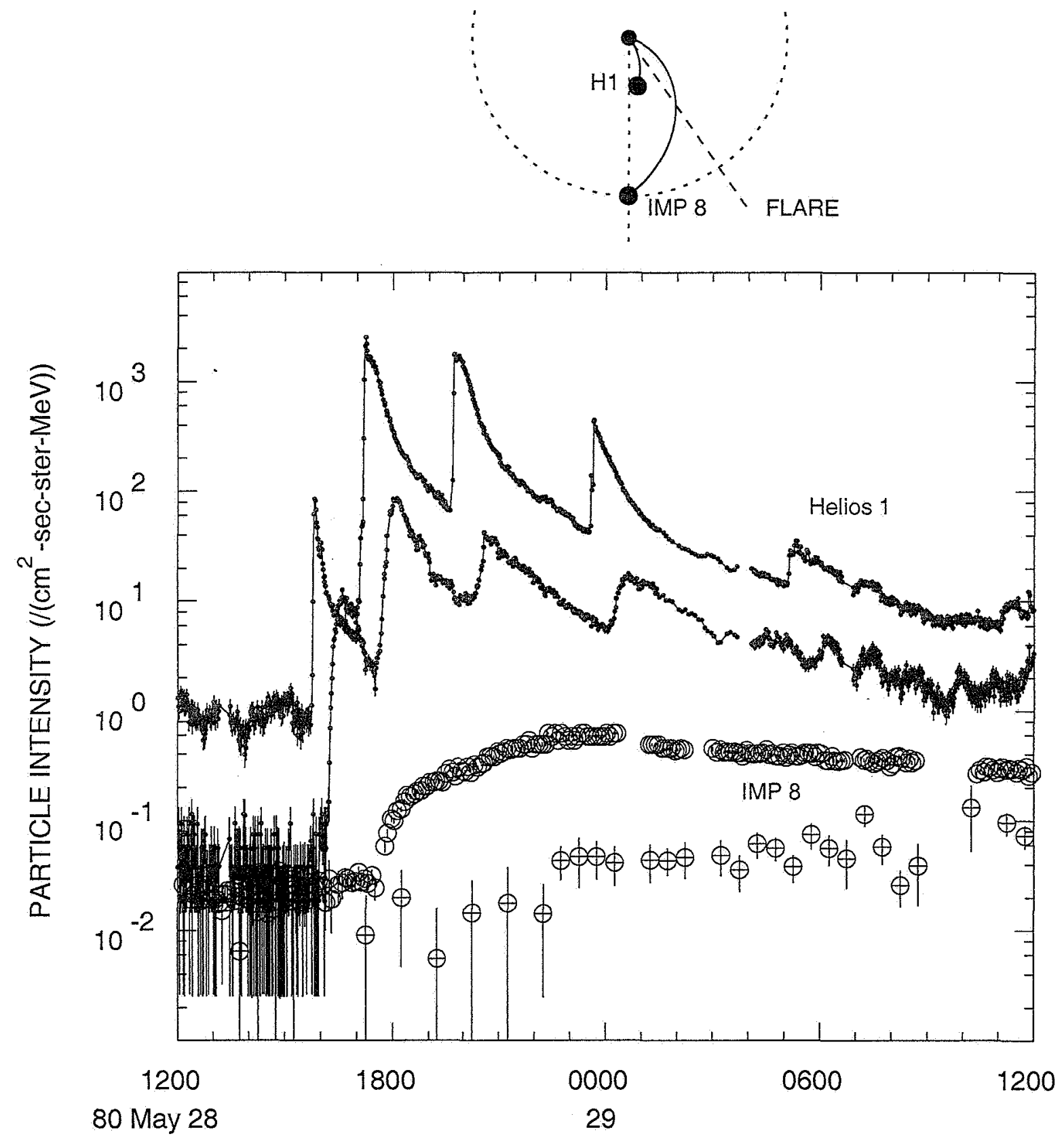

Fig. 3.- Electrons (first and third curves from top) and $2-4 \mathrm{MeV} / \mathrm{nuc}$ He ions (second and fourth curves from top) at Helios 1 and at IMP 8. The upper plot shows the location of the spacecraft and the flaring region. IMP 8 was more poorly connected to the flare $\left(\Delta=-46^{\circ}\right)$ than Helios 1 at 0.31 AU $\left(\Delta=-6^{\circ}\right)$. At IMP 8 interplanetary scattering plays a strong role so that the individual injections are not resolved. 

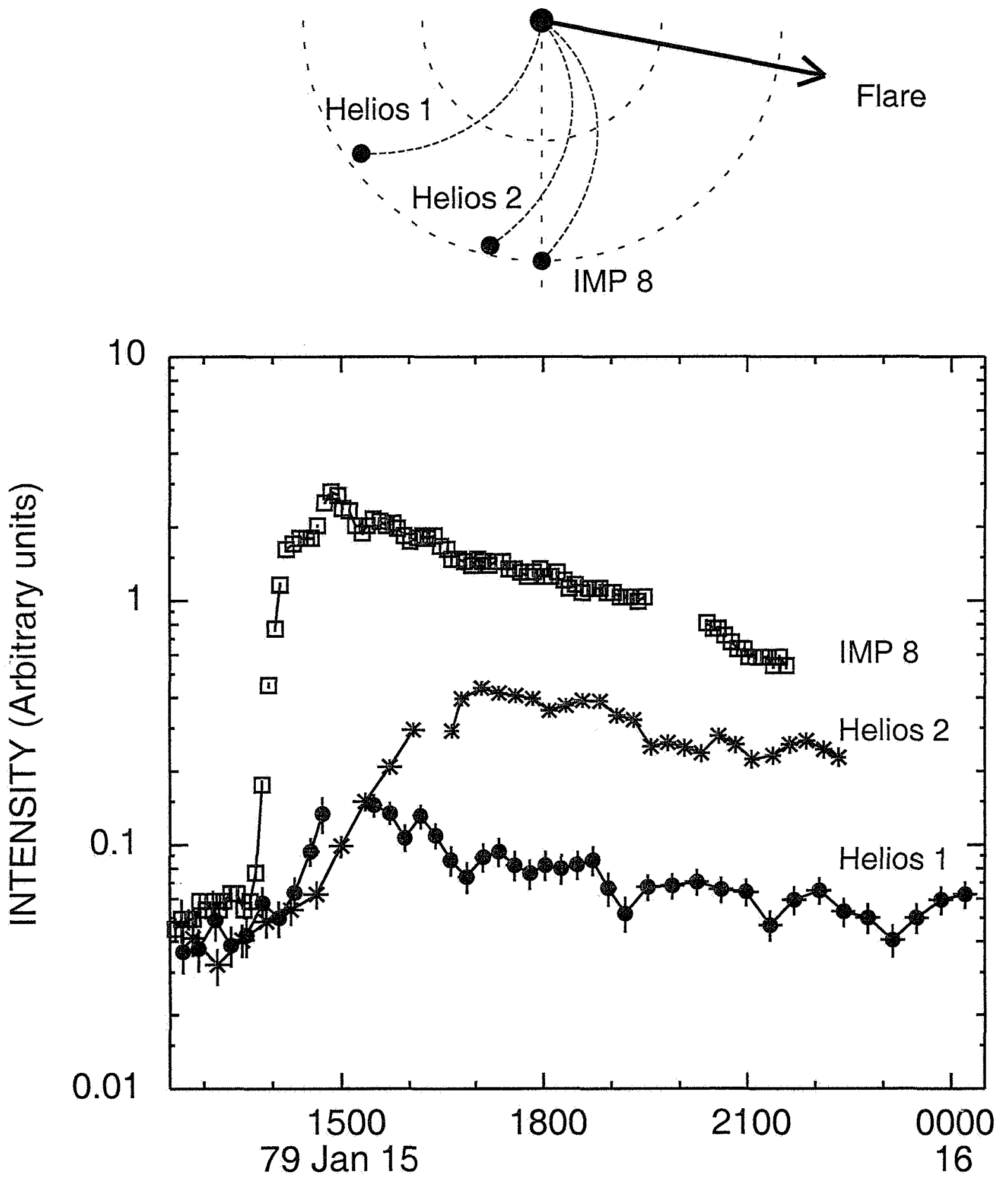

Fig. 4.- Connection plot and electron time profiles for the flare event of 1979 Jan 15. All radial positions are close to $1 \mathrm{AU}$, the connection angles $\Delta$ for the three positions vary from $31^{\circ}$ to $90^{\circ}$. The onset times and times-to-maximum are not correlated with the longitudinal distance. 

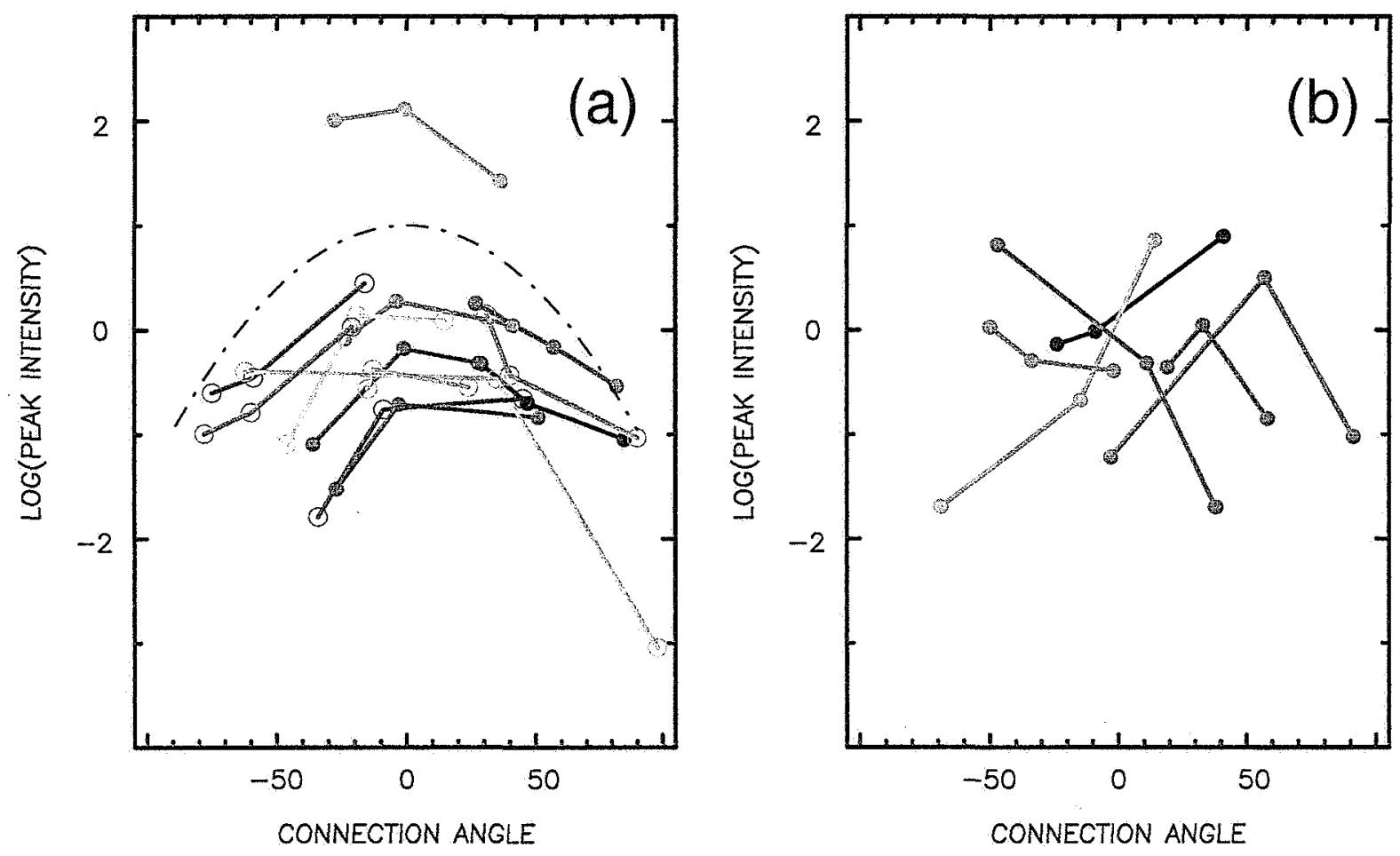

Fig. 5.- Variation of peak intensities $I_{m}$ with connection angle $\Delta$ for 19 events. Amplitudes for the same event are marked by the same symbols and colors and connected by straight lines. Amplitudes for distances different from 1 AU were corrected according to an $r^{-3}$-law. (a) events in which the largest amplitude occurs for the best connected spacecraft. The dashdotted line is a Gaussian distribution centered at zero longitude, with a standard deviation $\sigma=30^{\circ}$. (b) events with unusual variation of amplitudes with connection angle. 


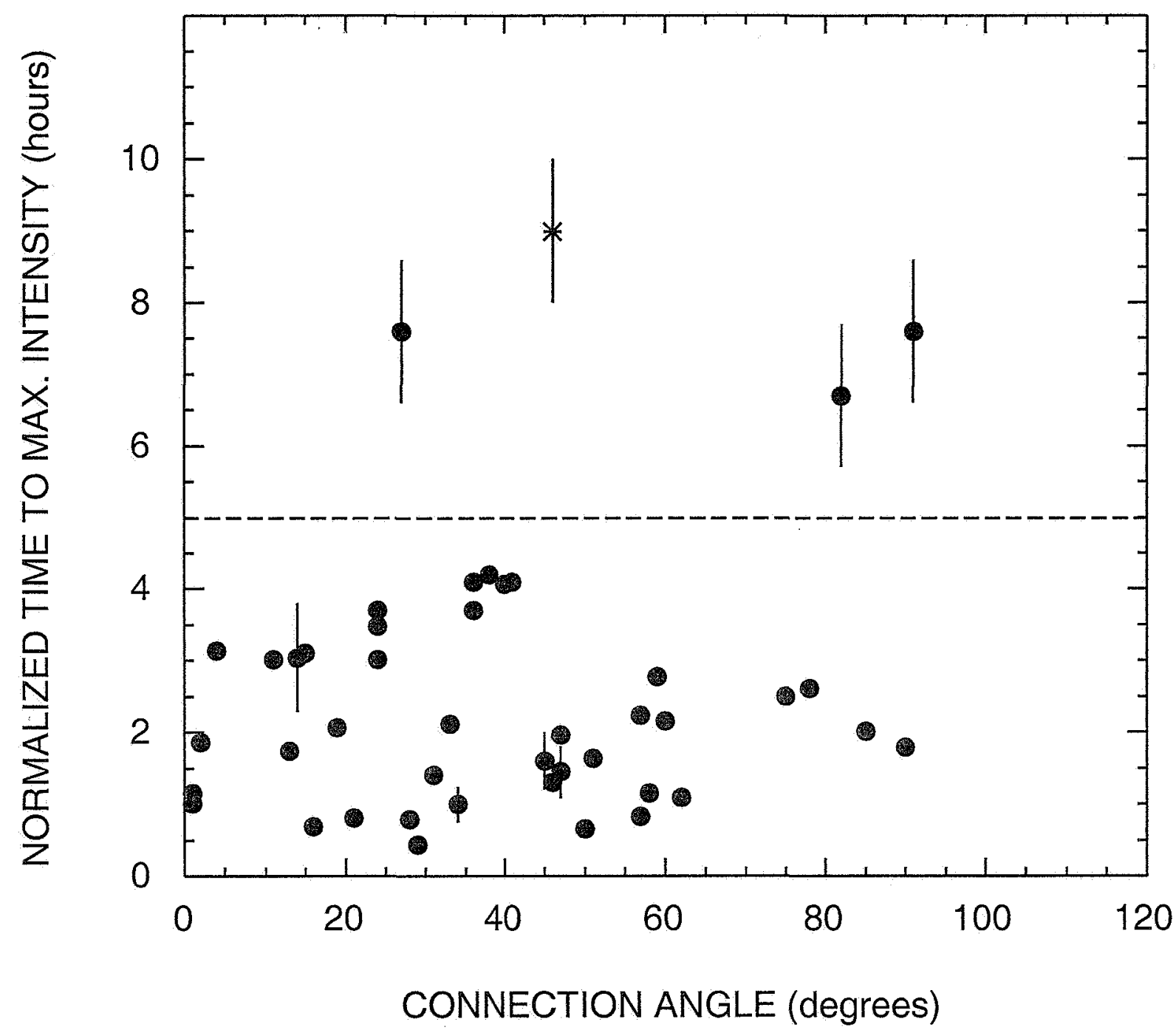

Fig. 6.- Normalized values of the time-to-maximum as a function of the absolute value of the angular distance $\Delta$. Radial distances are restricted to $r>0.6 \mathrm{AU}$. The normalization of the $t_{m}$-values was performed with an $r^{2-b}$-law, taking $b=0.5$. The uncertainty in $t_{m}$ caused by the value of $b$, if variable between 0 and 1 , is restricted to $25 \%$. This estimated error is shown for four examples with radial distances between 0.6 and $0.75 \mathrm{AU}$. The horizontal line at $5 \mathrm{hrs}$ would correspond to $\lambda_{0}=0.019 \mathrm{AU}$ in case of scattering along the average magnetic field. The four data points above this line are tentatively interpreted in terms of lateral interplanetary transport. 


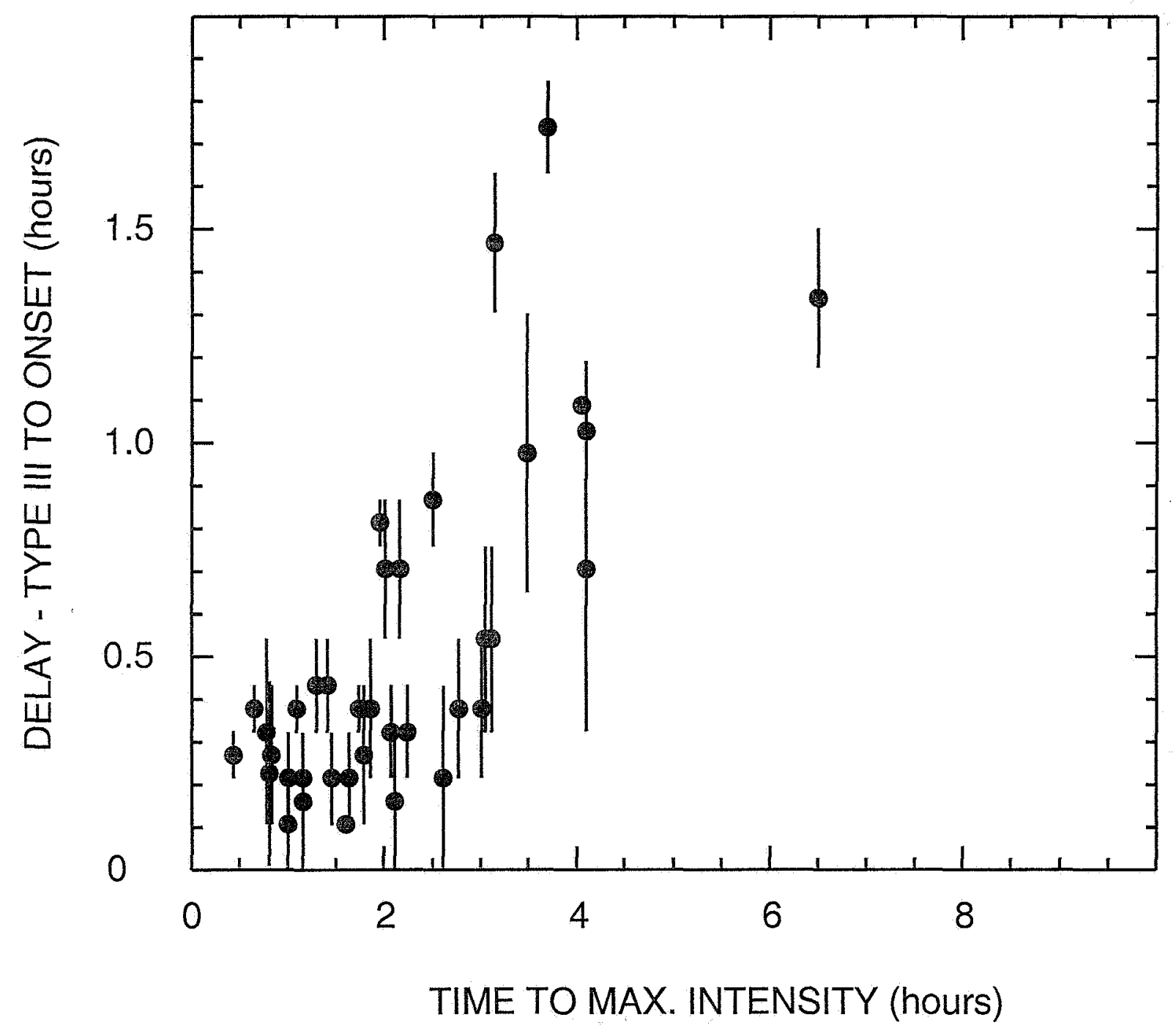

Fig. 7. - The onset times are plotted against the maximum times and found to be correlated. This indicates that the time when the intensity increases above background, marking the onset of an event, is related to the degree of interplanetary scattering. 

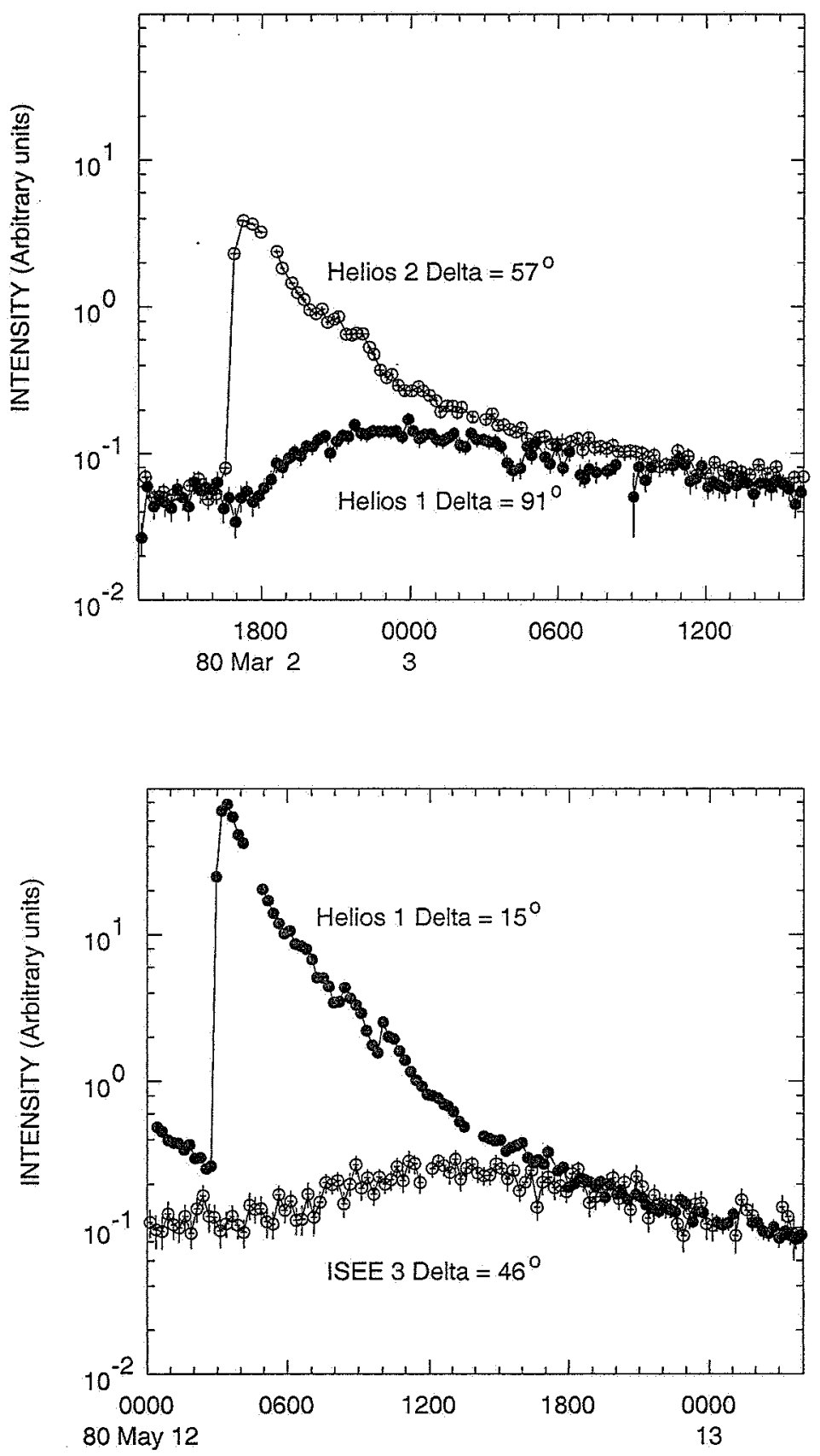

Fig. 8.- Electron time profiles for two flare events with unusually broad time profiles at one position. (a) For the event of $1980 \mathrm{March} 2 \mathrm{H} 1$ is at $r=0.98 \mathrm{AU}$ and a connection angle of $91^{\circ}$. (b) For the event of 1980 May 12 ISEE-3 is at a connection angle of $46^{\circ}$. The values of the radial mean free path estimated from the broad profiles would have been $\lambda_{o}=0.013 \mathrm{AU}$ (case (a)) and $\lambda_{0}=0.011 \mathrm{AU}$ (case (b)) in case of radial diffusion, below the range which has been assigned previously to scattering along the average IMF. We tentatively interpret this result in terms of transport processes perpendicular to the IMF direction. 